\title{
Les géographes - climatologues français et le changement climatique aux échelles régionales
}

\author{
Gérard Beltrando
}

\section{OpenEdition}

Journals

Édition électronique

URL : http://journals.openedition.org/echogeo/11816

DOI : 10.4000/echogeo.11816

ISSN : 1963-1197

Éditeur

Pôle de recherche pour l'organisation et la diffusion de l'information géographique (CNRS UMR 8586)

Référence électronique

Gérard Beltrando, "Les géographes - climatologues français et le changement climatique aux échelles régionales », EchoGéo [En ligne], Sur le Vif, mis en ligne le 12 avril 2010, consulté le 02 mai 2019. URL : http://journals.openedition.org/echogeo/11816 ; DOI : 10.4000/echogeo.11816

Ce document a été généré automatiquement le 2 mai 2019.

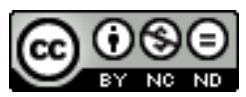

EchoGéo est mis à disposition selon les termes de la licence Creative Commons Attribution - Pas d'Utilisation Commerciale - Pas de Modification 4.0 International 


\title{
Les géographes - climatologues français et le changement climatique aux échelles régionales
}

\author{
Gérard Beltrando
}

\section{NOTE DE L'ÉDITEUR}

Un texte sur la conférence de Copenhague et le changement climatique avait été publié en janvier sur EchoGéo. Cette question fait l'objet de nombreux débats et prises de positions. Aussi EchoGéo vous propose-t-il un autre article sur le sujet.

\section{Introduction}

1 Depuis plus de 20 ans, les physiciens de l'atmosphère, relayés dans plusieurs pays par les mouvements écologistes, informent sur le risque pour la société des conséquences sociales et économiques d'un probable changement climatique dont l'origine pourrait être en grande partie le fait de l'Homme par l'intermédiaire des GES qu'il rejette massivement depuis plusieurs décennies ${ }^{1}$. En 2006, l'économiste N. Stern, a publié un rapport dans lequel il alerte sur les risques d'une récession économique « d'une ampleur catastrophique si rien n'était fait rapidement à l'échelle de la planète » et il indique que 1/100 du PIB investi maintenant suffirait à fortement atténuer les effets du changement climatique... Depuis la publication de ce rapport, largement médiatisé, plusieurs dirigeants politiques commencent à prendre conscience de l'existence de la responsabilité humaine dans ces dérèglements du climat et ils affichent leur intérêt pour ce nouveau sujet d'inquiétude pour la société. Timidement, plusieurs pays ont initié une politique de lutte pour réduire les rejets de GES marquant ainsi un modeste changement de cap. Mais la récente conférence de Copenhague, qui s'est achevée par la publication d'un document non contraignant pour les pays émetteurs, est bien en deçà des volontés 
affichées. Ce document, qui ne comporte aucun engagement chiffré de réduction des émissions, montre aussi les divisions entre les pays industrialisés, peu enclins à remettre en cause leur modèle de croissance, et les pays du Sud, inquiets pour leurs projets de développement et qui souhaitent bien naturellement imiter les pays riches.

Souvent loin de ces négociations politiques, de nombreux scientifiques, d'horizons disciplinaires différents, observent et analysent les indicateurs du changement climatique en cours alors que d'autres s'intéressent à l'adaptation des sociétés à un climat qui pourrait être différent de l'actuel. En France, quelques géographes spécialisés dans les questions environnementales participent à certains de ces travaux. Le présent document évoque des publications de quelques uns d'entre eux qui travaillent sur le constat des décennies qui viennent de s'écouler, à travers l'étude des séries climatiques ou des indicateurs de ces changements (dynamique des glaciers, phénologie végétale, trait de côte...). Mais, devant la complexité des relations entre l'Homme et la Nature (nombreuses rétroactions sur les milieux physiques des actions de l'Homme) et peut-être aussi devant la part d'incertitude intrinsèque aux résultats fournis par les modèles numériques et reconnue par les physiciens eux même ${ }^{2}$, peu de travaux de géographes français ont été consacrés à l'analyse des données produites par les modèles numériques du climat, pour évaluer les répercussions que pourraient avoir sur les hommes ces changements tels qu'ils sont simulés par ces modèles.

\section{Une certitude : la tendance globale est à l'augmentation des températures et des gaz à effet de serre}

Le changement climatique, déjà perceptible sur de nombreux indicateurs des milieux physiques, coïncide avec une augmentation importante des GES et des aérosols que l'Homme injecte dans l'atmosphère ${ }^{3}$. Ces GES additionnels s'ajoutent à ceux qui sont naturellement présents et permettent un " effet de serre naturel », sans lequel la vie sur terre ne serait pas possible sous la forme que nous connaissons. C'est l'addition de ces GES d'origine anthropique qui constitue ce que l'on appelle "l'effet de serre additionnel", phénomène qui pour la grande majorité des scientifiques explique l'essentiel de la tendance pluriannuelle positive au réchauffement observée depuis quelques décennies. Ce qui n'exclut pas l'existence de périodes avec une tendance négative résultant d'interaction au sein du système climatique planétaire (cf. par exemple le cas de l'Arctique évoqué au § 2.2).

\subsection{Les tendances observées}

Depuis 150 ans, l'utilisation des combustibles fossiles est largement responsable de l'augmentation d'environ un tiers de la concentration en gaz carbonique $\left(\mathrm{CO}_{2}\right)$ (figure 1). 
Figure 1 - Température (en ${ }^{\circ} \mathrm{C}$ ) et taux de $\mathrm{CO} 2$ (en partie par million de volume, ppmv) - Valeurs annuelles moyennes de 1860 à 2008

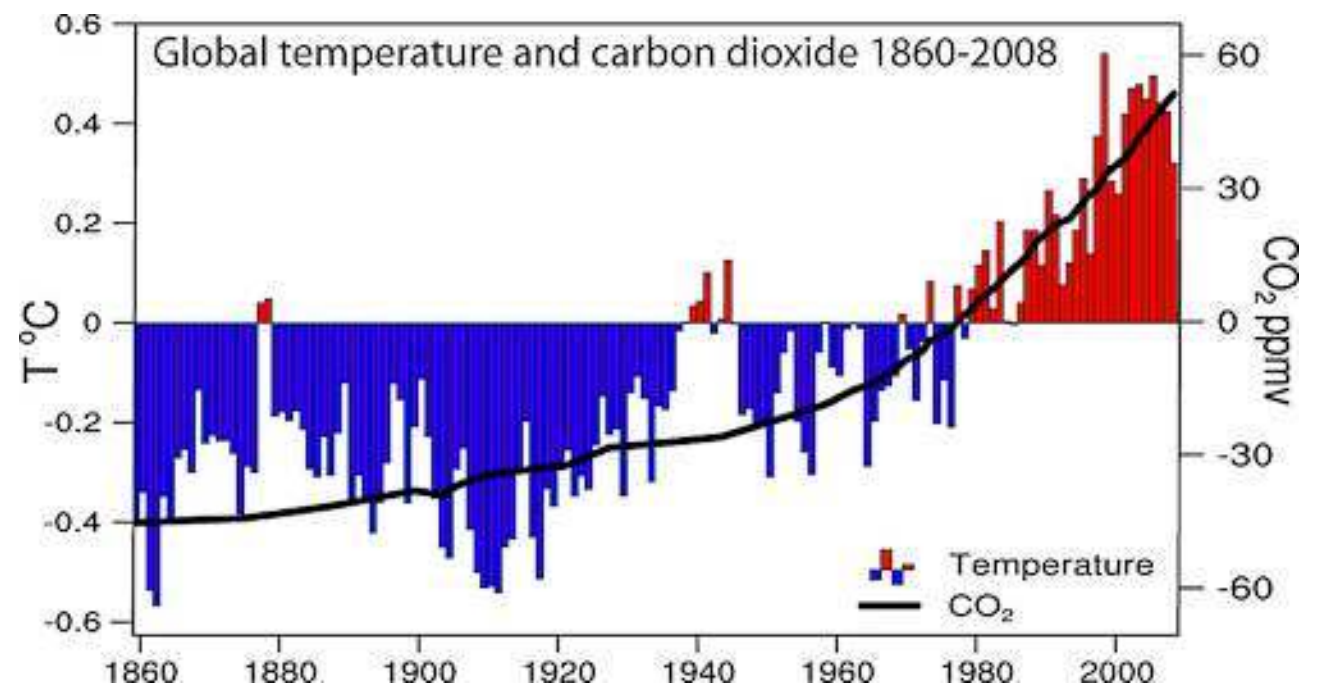

Source : d'après Trenberth 2009, site du Carbon Dioxyde Information Analysis centre

5 Le carbone injecté joue un rôle essentiel dans l'effet de serre additionnel car si une partie est absorbée par les océans et la biosphère continentale, le reste s'accumule dans l'atmosphère. C'est cette modification de la composition de l'air sous l'action des activités humaines qui perturbe l'équilibre énergétique de la planète.

6 Sur la figure 2 est indiquée la part respective, en $\mathrm{W} / \mathrm{m}^{2}$, des paramètres d'origine anthropique qui contribuent à modifier les échanges radiatifs depuis le début de l'ère industrielle. 
Figure 2 - Moyenne mondiale des composantes du forçage radioactif en 2000 par rapport à 1750

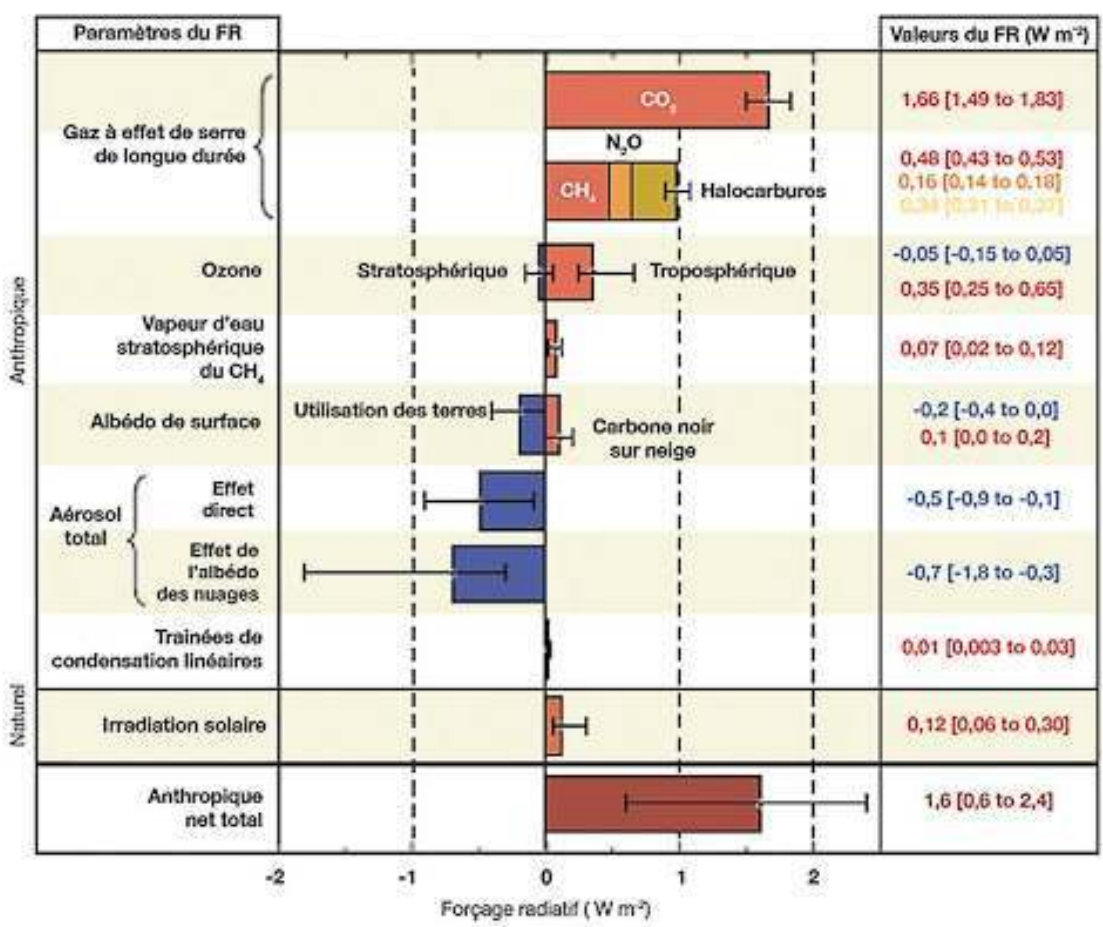

Les barres noires horizontales donnent l'intervalle de confiance accordée par les scientifiques. La vapeur d'eau (H20), premier GES, mais dont la concentration est peu influencée par les activités humaines, n'est pas indiquée sur cette figure.

Source : IPCC, 2007 http://adaptation.nrcan.gc.ca/assess/2007/ch2/images/fig4_f.jpg

Ces forçages radiatifs (FR) peuvent être, soit positifs soit négatifs :

- A gauche de la ligne verticale « 0 » apparaissent les paramètres qui contribuent à un « forçage radiatif négatif » (c'est-à-dire qui contribuent au refroidissement de la basse atmosphère). Cela concerne surtout l'albédo (réflexion), liée à l'extension des surfaces urbanisées et agricoles ${ }^{4}$, l'augmentation du taux d'aérosols et de la couverture nuageuse ${ }^{5}$. Mais pour ces deux derniers paramètres, la marge d'incertitude est relativement importante compte tenu de l'état de la science : il est par exemple difficile d'évaluer la nébulosité pour la période antérieure à celle couverte par les satellites d'observation....

- A droite de la ligne verticale « 0 » apparaissent les facteurs qui contribuent à un « forçage radiatif positif » (réchauffement). Le $\mathrm{CO}_{2}$ joue le rôle principal dans ce forçage, avec quelques autres gaz, également à structure au moins triatomique : Méthane $\left(\mathrm{CH}_{4}\right)$, Protoxyde d'azote $\left(\mathrm{N}_{2} 0\right)$, Ozone de la troposphère $\left(\mathrm{O}_{3}\right)$, gaz artificiels fabriqués par l'homme, tels les composés chimiques commercialement appelés Fréon et divers gaz industriels... Ils absorbent plus fortement le rayonnement tellurique (grandes longueurs d'onde) émis par la surface de la planète que le rayonnement solaire (courte longueur d'onde). Ce sont ces gaz, dont la concentration a fortement augmenté depuis le début de l'ère industrielle, qui expliquent l'effet de serre additionnel.

Le $\mathrm{CO} 2$ joue donc un rôle majeur dans l'effet de serre additionnel, mais il n'est pas le seul en cause: la teneur en méthane ( $\mathrm{CH} 4)$ a plus que doublé, sur la même période, essentiellement à cause du développement de l'agriculture (fermentation anaérobie des ruminants, riziculture inondée...) et une bonne part de l'accroissement d'environ $15 \%$ de 
la teneur en protoxyde d'azote (N2O) s'explique par la combustion des combustibles fossiles ainsi que la fertilisation azotée des sols (IPCC, 2007). Ces deux GES (CH4 et N2O), bien que présents en quantité sensiblement plus faible que le $\mathrm{CO} 2$, ont un pouvoir de réchauffement global (PRG) nettement supérieur. Par exemple, sur une période de 100 ans, un $\mathrm{kg}$ de $\mathrm{CH} 4$ dans l'atmosphère a un impact sur l'effet de serre, 23 fois plus fort qu'un kilo de CO2..., (IPCC, 2007). A côté des GES "naturels", il en existe de nombreux autres, qualifiés ici d'"artificiels" (halocarbures, Fréon...) qui sont présents en quantité encore plus faible mais qui ont un PRG très nettement supérieur à l'ensemble des GES d'origine naturelle.

Le bilan de ces forçages, négatifs et positifs, d'origine humaine est estimé à $+1,6 \mathrm{~W} / \mathrm{m}^{2}$ en 2000 par rapport à 1750 (figure 2). A ce bilan, il faut ajouter $+0,12 \mathrm{~W} / \mathrm{m}^{2}$ qui proviennent de l'augmentation de l'irradiation solaire (phénomène également naturel).

En parallèle et sur la même période, la température moyenne de l'air a augmenté d'environ $0,8^{\circ} \mathrm{C}$ dont $0,6^{\circ} \mathrm{C}$ durant le XXe siècle (cf. figure 1). Mais cette tendance globale des températures n'est pas linéaire et elle se manifeste par une assez grande variabilité pouvant se traduire localement par des tendances négatives sur des périodes plus ou moins longues. Cela est tout à fait cohérent compte tenu de la multiplicité des interactions internes au système climatique : la vague de froid que vient de connaître l'Europe durant l'automne et l'hiver 2009-2010 peut parfaitement s'inscrire dans cette tendance au réchauffement car la variabilité naturelle du temps et du climat reste une caractéristique normale et elle pourrait même être amplifiée dans le futur d'après les résultats des simulations climatiques. Reste bien évidemment l'interrogation sur la part que pourrait représenter la tendance au réchauffement observé depuis quelques décennies par rapport à la sortie du Petit Age Glaciaire (maximum durant la deuxième moitié du $\mathrm{XV}^{\mathrm{e}}$ siècle), considéré comme l'épisode le plus froid de l'Holocène supérieur (Mann and al., 1998) et qui peut aussi expliquer une part de la tendance actuelle. Cela ne doit pas constituer un frein aux études sur le changement climatique d'origine anthropique qui demandent la meilleure anticipation possible des sociétés.

11 Grâce à l'évolution technologique, permettant de mesurer plus finement les concentrations en GES et les aérosols et aux campagnes de mesures qui se sont multipliées depuis quelques décennies, il existe un nombre important d'indicateurs (thermiques, hydrologiques, biologiques...) qui confirment ces changements dans les milieux physiques.

\subsection{Les indicateurs du changement}

Même si le changement climatique contemporain, et quel qu'en soit son origine, n'est pas le seul responsable des désordres environnementaux observés, il existe de nombreux indicateurs de ce changement qui affecte, à des degrés divers, la plupart des régions du monde ${ }^{6}$.

Parmi les indicateurs, très largement médiatisés, on peut citer :

- un réchauffement superficiel des océans qui, à eux seuls, absorbent plus de $80 \%$ de la chaleur ajoutée au système climatique, ce qui se traduit par une élévation moyenne à l'échelle globale de près de $8 \mathrm{~cm}$ entre 1961 et 2003, pour un quart environ par dilatation thermique (Lévitus and al., 2005). D'un océan à l'autre, le niveau varie aussi sous l'effet 
d'autres facteurs tel que le phénomène couplé appelé El Niño/Oscillation Australe pour le Pacifique, décrit depuis près de 30 ans (Rasmusson and Carpenter, 1982)

- une fonte de la banquise qui modifie la réflexion du rayonnement solaire et permet à la planète d'absorber plus d'énergie solaire (Le Clainche and al., 2001): ainsi laglace de mer de l'Arctique décline actuellement à un taux de $11 \%$ par décennie, par rapport à la moyenne de 1979 à 2000 (site web duNational Snow and Ice Data Center)

- un bilan de masse négatif des inlandsis et de la plupart des glaciers de montagne. Les reculs de la période en cours sont le résultat d'une augmentation très importante de la fusion estivale non compensée par les apports en eau (Lombard, 2005)

- un changement des stades phénologiques de la végétation naturelle ou cultivée. Ainsi dans la quasi-totalité des vignobles français, depuis deux décennies les vendanges ont lieu plus tôt que dans les décennies précédentes, les rendements agronomiques et le taux d'alcool ont augmenté (cf. § 2.1). Certes, l'évolution des techniques de conduite agronomiques intervient dans ces tendances, mais lorsque cela est le cas, elle se traduit plutôt par une rupture dans le changement ; de même le taux d'alcool varie d'une année à l'autre ce qui n'est pas le cas des techniques agronomiques

- etc...

Il y a donc bien, depuis quelques décennies, de nombreux indicateurs de changements dans les milieux physiques qui sont manifestement en phase avec une évolution du climat.

\section{Le constat du changement climatique observé : un domaine d'étude bien connu des géographes}

L'analyse des séries climatiques est l'un des tout premiers sujets d'étude des géographesclimatologues ${ }^{7}$. Ces travaux permettent de préciser la variabilité spatiale et temporelle du climat ou encore de montrer que les changements ne touchent pas de la même manière les températures moyennes, maximales et minimales d'une même station. Ainsi, Douguédroit et Bridier (2008) montrent que l'évolution des températures dans le sud-est de la France, au cours de la période 1951-2007, n'est pas un phénomène uniforme, ni dans l'espace, ni dans le temps. Le réchauffement touche aussi différemment les stations représentatives des environnements ruraux, où les transferts de chaleur latente sont généralement plus importants, que celles représentatives des environnements urbains au bâti dense et où les transferts de chaleur latente sont la plupart du temps moins importants qu'en zone rurale.

Mais une « rupture » statistique apparait à la fin des années 1970/début des années 1980 dans les séries chronologiques de nombreuses variables climatiques et hydrologiques. Cette période marque le début d'une tendance à la hausse des températures dans de nombreuses régions du globe. Les quelques exemples ci-dessous sur les vignobles puis sur la couverture neigeuse et glaciaire illustrent ces démarches.

\subsection{Variabilité temporelle du climat et relation avec la phénologie de la vigne}

17 Déjà en 1996, V. Bonnardot montrait les relations significatives entre les dates des stades phénologiques et les températures dans le vignoble de Bourgogne. Toujours pour ce 
vignoble, Chabin et al. (2007), à partir des données climatiques et phénologiques de la vigne, ont comparé deux sites de la région de Beaune : l'un sur la Côte, l'autre dans les Hautes Côtes (La Rochepot). Ces auteurs montrent que l'augmentation des températures, depuis 1987-1988, explique une précocité relative des stades phénologiques et un raccourcissement de la période débourrement-maturité. Cela se traduit par des vendanges plus précoces et la multiplication des «bons millésimes». Cela pourra aussi progressivement être à l'origine d'une possible remise en cause (observée en 2003) de la typicité traditionnelle du vin, voire de la hiérarchie qualitative de ces vignobles, car ce réchauffement de l'air a plus d'effets bénéfiques sur les vignobles implantés relativement haut sur l'arrière-côte que sur ceux implantés à une altitude plus faible (les plus célèbres...) (figure 3). Un tel constat n'est pas sans conséquence, surtout en France, compte tenu du poids culturel et économique que représente la viti-viniculture et de l'importance particulière accordée à la notion de « terroir ».

Figure 3 - Relations entre températures moyennes (avril - septembre) et dates de maturité vendanges du Pinot noir à Beaune et à la Rochepot

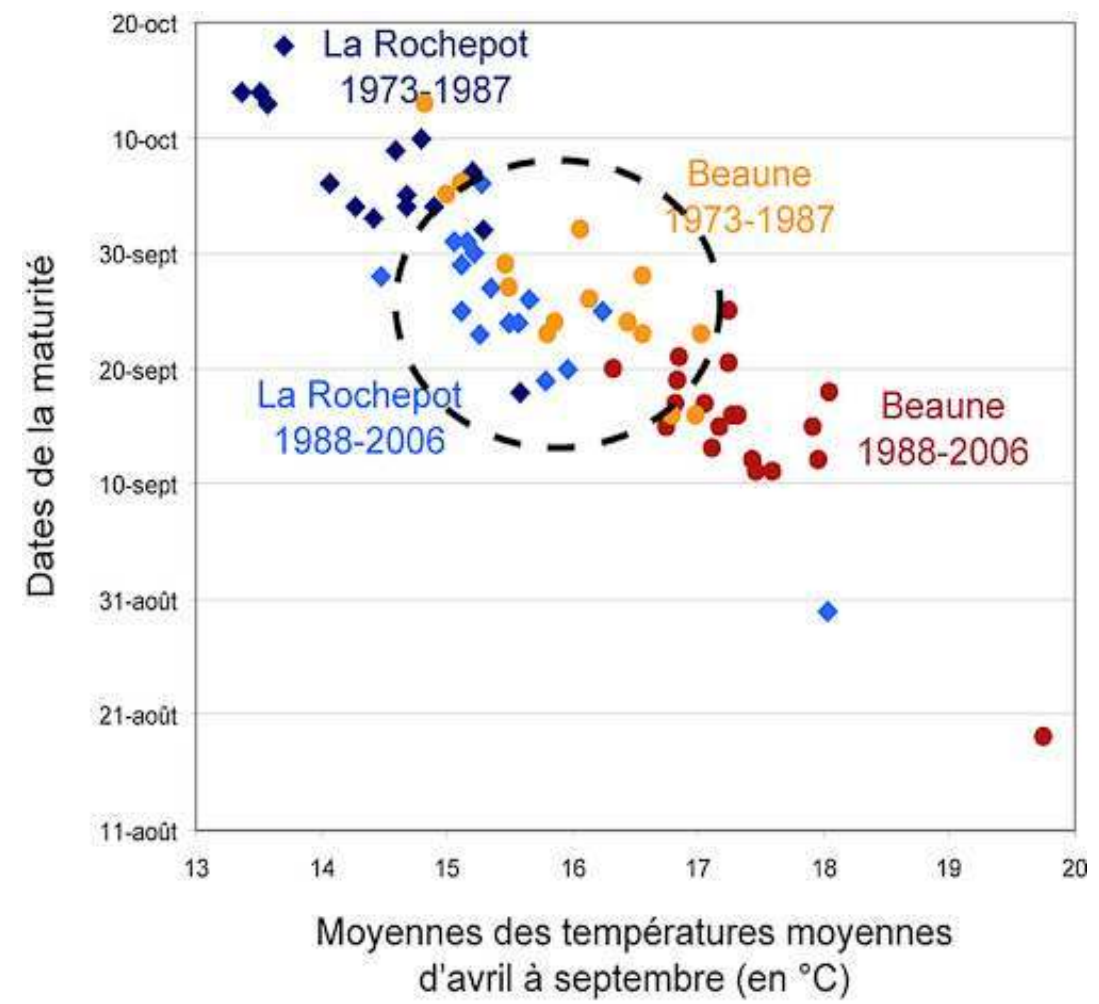

Depuis 1988, les températures moyennes de la Haute Côte sont comparables à celles de Beaune entre 1973 et 1987

Source : Chabin et al., 2007

18 La modification des dates d'apparition des stades phénologiques en liaison avec l'augmentation des températures est générale dans les vignobles et en particulier en France. Ce thème intéresse les géographes mais aussi les agronomes qui parfois s'associent sur des programmes communs : travaux sur les Côtes du Rhône et les Côtes de Provence (Seguin, 2006, Bélia et al., 2008), le Bordelais (Bois, 2007), l'Alsace (Duchesne et Schneider, 2007), le Val de Loire (Bonnefoy et al., 2009), la Champagne (Briche et al., 2009)... Partout les travaux montrent une avancée des stades phénologiques au moins après le débourrement et ce constat dépasse largement le cadre du territoire français 
comme l'ont constaté par exemple Jones et al. (2005) sur plusieurs régions viticoles européennes. Cette évolution est aussi constatée pour d'autres cultures (blé, maïs, arbres fruitiers...) ainsi que d'autres formations herbacées et arbustives (Walther and al., 2005).

\subsection{Le changement climatique et la dynamique des glaciers et de la neige}

19 La dynamique de la couverture neigeuse et glaciaire est aussi un sujet de prédilection des chercheurs qui ont souvent pour objectif de décrire et d'expliquer la variabilité des écoulements liquides ou de l'enneigement. Par exemple, Loubier (2007) montre, pour les domaines skiables de Savoie et de Haute-Savoie, que le changement climatique posera des problèmes d'exploitation des pistes en début et en fin de saison et que la multiplication des canons produisant de la neige « de culture » aurait des retombées environnementales et génèrerait des conflits d'intérêt entre les utilisateurs de l'eau. Dans d'autres régions du monde, par exemple les déserts centrasiatiques, où l'agriculture oasienne est dépendante de l'eau des hauts massifs, la baisse du stock de glace aura des répercussions sociales et économiques nettement plus dramatiques...

Les géographes travaillent aussi sur l'évolution des glaciers dont le bilan de masse est négatif dans la plupart des régions alors qu'il est positif dans quelques unes ${ }^{8}$. Ainsi, Chenet et Roussel (2008) montrent que le changement climatique observé depuis la fin du Petit Age glaciaire en Islande entraîne un retrait généralisé des fronts glaciaires en phase avec une élévation des températures qui est plus marquée sous les hautes latitudes qu'ailleurs sur la planète' 9

21 De même, Cossaert et Le Gall (2008) observent un important recul des glaciers du massif de l'Acongua depuis la fin du Petit Âge glaciaire. Si ces glaciers disparaissent, ils entraineront une modification des régimes hydrologiques qui, jusqu'à présent, se caractérisaient par de hautes eaux estivales nécessaires à l'agriculture irriguée du piémont des Andes (figure 4). Ce pic estival pourrait à l'avenir être atténué et remettre en cause les stratégies de gestion de la ressource en eau. Les études géomorphologiques permettent aussi de repérer les zones déjà vulnérables qui pourraient l'être encore plus dans le futur. Fort et al. (2009) montrent que certains systèmes géomorphologiques (barrage, rétention de sédiments...) pourront, dans un contexte de changement climatique, amplifier la menace qu'ils font déjà peser sur les zones habitées en contrebas. 


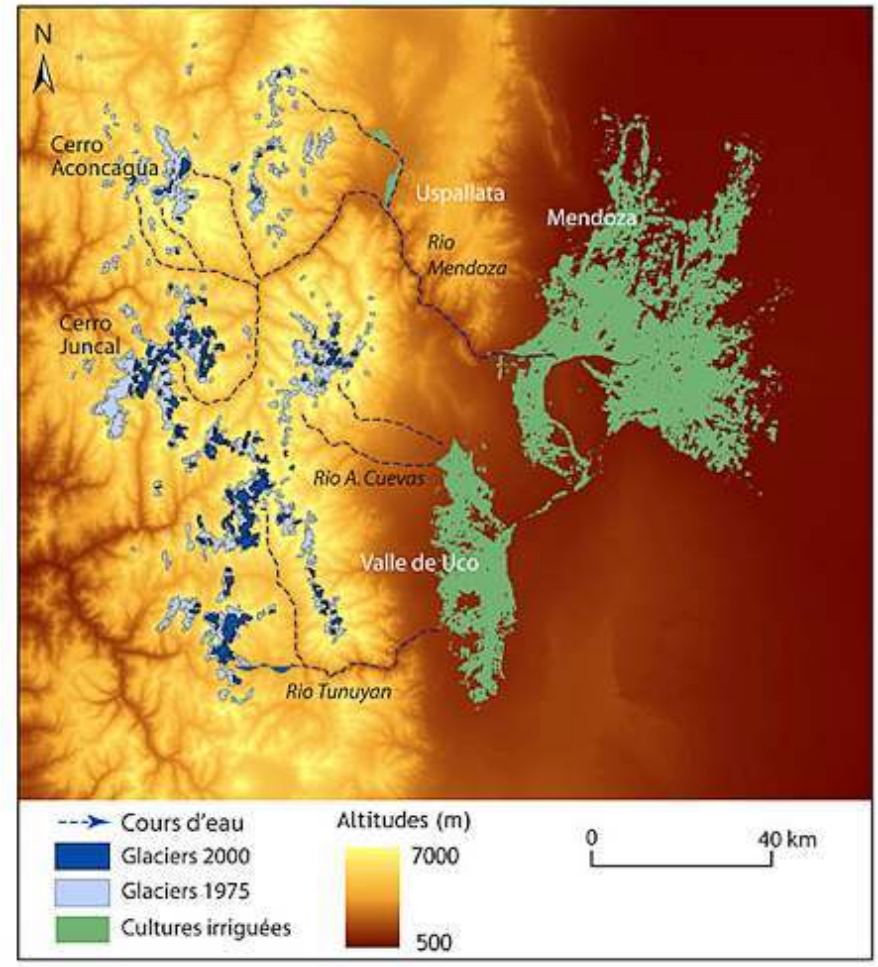

Source : d'après Cossart et Le Gall, 2008. Document obtenu à partir de la signature spectrale des glaciers, validée par des relevés terrains réalisés par d'autres auteurs http://echogeo.revues.org/ index2416.html

Ces études sur le changement climatique peuvent aussi être l'occasion de mieux décrire la mosaïque des climats qui caractérise les régions de montagne où la topographie et l'exposition aux flux atmosphériques introduisent une grande diversité climatique qui n'est pas toujours connue avec une précision suffisante. Pour cela, des campagnes de mesures avec des matériels de mesures plus ou moins sophistiqués peuvent être nécessaires. Ainsi, pour établir un diagnostic du climat à l'échelle de la réserve des Hauts Plateaux du Vercors, Bigot et al. (2006) ont installé un ensemble d'instruments de mesures avec notamment un capteur ultrasonique de hauteur de neige et des capteurs de rayonnement incident et réfléchi. Ailleurs dans le massif alpin, Bodin (2007) montre, à partir d'une analyse de la distribution spatiale du pergélisol et en élaborant un Modèle Numérique de Terrain (MNT), que le réchauffement climatique des dernières années semble avoir eu pour effet une accélération des vitesses de fluage du pergélisol, qui pose la question de la stabilité des versants gelés en cours de dégradation....

Pour l'Arctique, une des région-clé pour l'observation et la compréhension des variations climatiques, Kergomard (2005) montre que le réchauffement durant le XXe siècle est plus important que la moyenne du globe, mais comme ailleurs «ce réchauffement n'a été ni continu dans le temps, ni homogène dans l'espace ». En particulier, après une forte hausse des températures au début du siècle, une tendance à la baisse est constatée à partir des années 40 , qui s'est poursuivie plus ou moins tardivement selon les secteurs. «Des cycles complexes associant les circulations atmosphérique et océanique sur l'Arctique et les processus d'échange thermodynamiques à l'interface océan-atmosphère (en y incluant le rôle de la banquise)... peuvent être associés aux mécanismes de l'Oscillation Nord Atlantique (ONA) et de 
l'Oscillation Arctique (OA)» (figure 5). La prise en compte de ces interactions permet de comprendre par exemple pourquoi dans un contexte de fort réchauffement, certaines parties de la surface du globe peuvent enregistrer un refroidissement. ${ }^{10}$

Figure 5 - Une représentation des interactions entre la variabilité atmosphérique (mécanisme de l'Oscillation Nord-Atlantique et de l'Oscillation Arctique), les glaces et les courants marins de l'Arctique

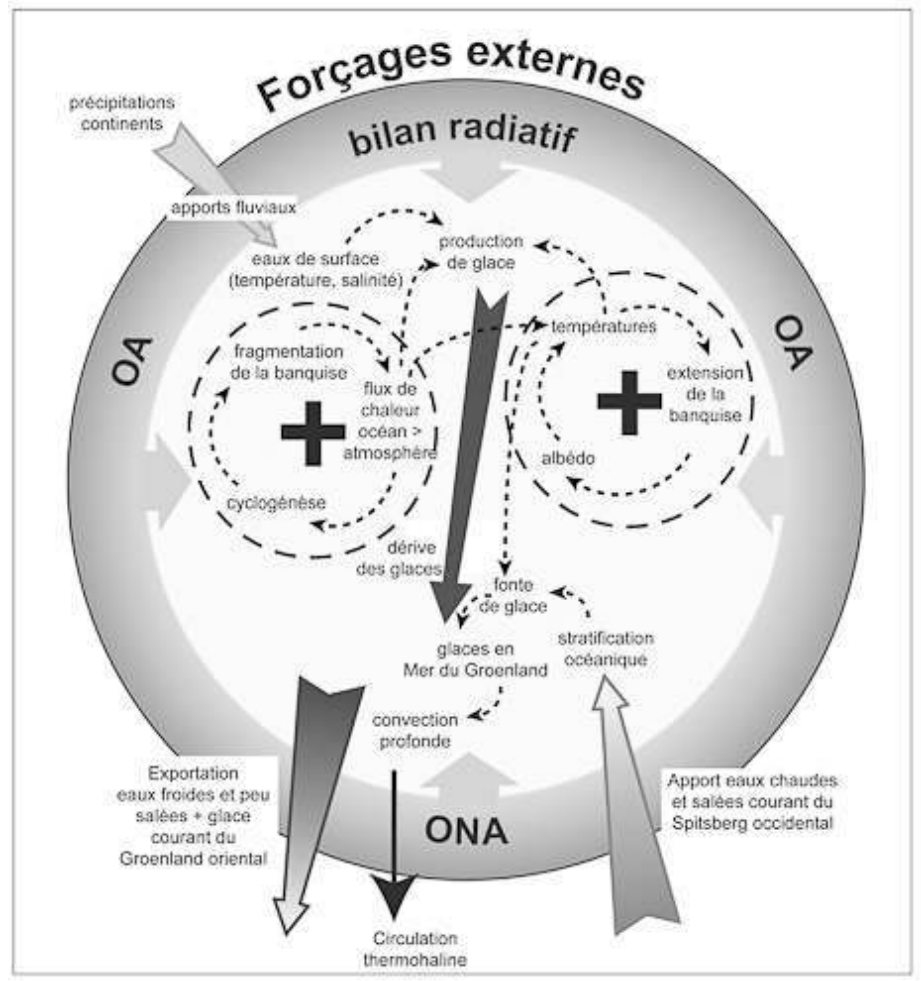

Source : Kergomard, 2005. Site web : http://134.59.38.8/ umr/climat/climat_societe/ Dijon2005/3CKcd.pdf

Ce dernier exemple illustre aussi un autre type de démarche bien employée par les géographes-climatologues : celle qui consiste à établir des téléconnexions (relation entre des variables du système climatique) et à préciser le rôle des mécanismes couplés entre l'océan et l'atmosphère, dont le plus connu est le phénomène El Niňo-Oscillation Australe qui concerne les latitudes intertropicales du Pacifique. De nombreux travaux depuis une vingtaine d'années ont été consacrés à ce thème et à ses répercussions sur la pluviométrie et l'agriculture (Beltrando et Cadet, 1990 ; Beltrando and Camberlin, 1993...). Mais ce type de mécanisme océano-atmosphérique, même s'il a un lien avec l'effet de serre additionnel (eau superficielle plus chaude), reste un phénomène naturel caractérisé par sa propre variabilité. Ce qui bien évidemment introduit une source supplémentaire de complexité et trop souvent ce phénomène est associé de manière abusive au changement climatique. 


\section{La réticence des géographes sur la performance de la modélisation : une prudence compréhensible qui devrait s'atténuer}

climatologues utilisent des Modèles de Circulation Générale (MCG, appelés aussi GCM suivant l'abréviation anglophone) permettant de simuler « au mieux » le fonctionnement de l'atmosphère et l'évolution possible des paramètres du climat en fonction de divers scénarios d'évolution de la concentration en GES. Un MCG est donc un outil numérique qui résout les équations primitives de la mécanique et de la thermodynamique des fluides géophysiques dans l'espace ( $3 \mathrm{D}$ ) ainsi que dans le temps; les équations permettent aussi par exemple de simuler le transfert du rayonnement. Mais la résolution spatiale des modèles est limitée par la puissance des ordinateurs existants et par la précisions des données d'entrée du modèle; certains processus thermodynamiques d'échelle relativement réduite (turbulence, convection...) doivent être paramétrés et bien évidemment cela introduit des incertitudes.

\subsection{Une marge d'incertitude qui diminue sans être pour autant négligeable}

Plusieurs sources d'incertitude existent dans la simulation du futur de l'atmosphère : par exemple l'évolution temporelle des apports de rayonnement solaire, le rôle des nuages dans le bilan radiatif de la planète ou encore le traitement de la convection "humide" (avec changement de phase de l'eau et éventuellement formation des précipitations). Ces problèmes complexes exigent donc encore des recherches fondamentales. Ces incertitudes, tout à fait compréhensibles, sont à l'origine d'une imprécision dans les simulations et elles expliquent aussi le fait que les résultats des MCG sont donc variables d'un paramètre à l'autre. Par exemple, l'incertitude sur la prévision est plus importante pour la pluviométrie que pour les températures.

La modélisation numérique repose sur une division de l'atmosphère en un grand nombre de "volumes élémentaires", correspondant chacun à des "paramètres supposés homogènes » tels que la densité de l'air, le vent, la température, la pression, la nébulosité...Ces volumes sont empilés les uns sur les autres pour tenir compte de la structure verticale de l'atmosphère (figure 6). La nature de la surface des terres (biosphère notamment) est aussi prise en compte, mais parfois de manière très grossière ce qui introduit une nouvelle part d'incertitude.... 


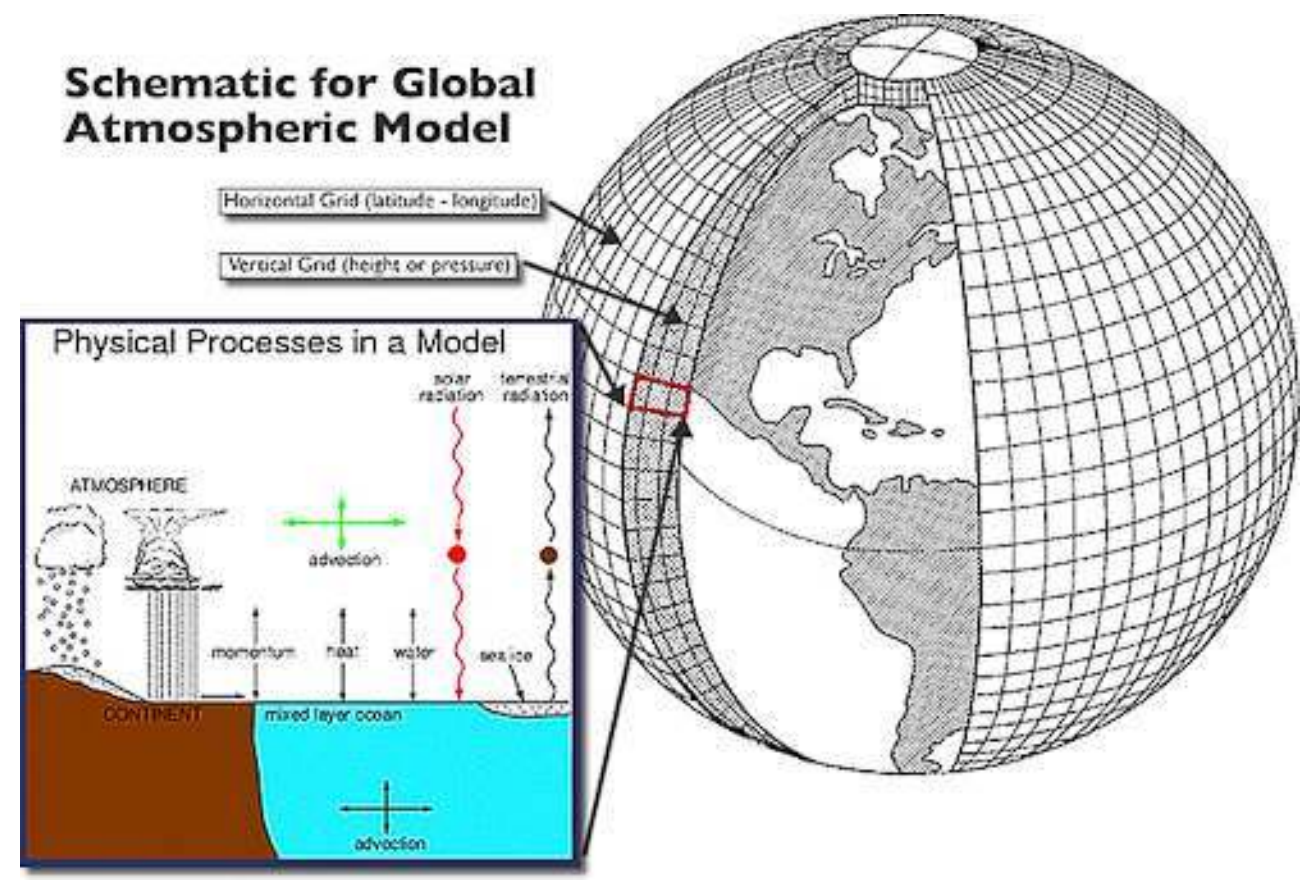

Source : Document NOAA, domaine public. Image disponible : http://en.wikipedia.org/wiki/ File:Global_Atmospheric_Model.jpg

Un MCG est donc la meilleure traduction numérique (ou la moins mauvaise) connue par les hommes, à un moment donné, du paramétrage des principaux processus physiques, géophysiques, chimique et biologique qui régissent le système climatique. Malgré la rigueur apportée par les scientifiques pour représenter avec le plus de réalisme possible l'énorme complexité de la physique atmosphérique, aucun d'entre eux ne peut simuler avec précision le comportement futur de l'atmosphère. Cela est bien connu des modélisateurs même s'ils ne le mettent pas toujours en avant, notamment lorsqu'il s'agit de communiquer des résultats aux décideurs (peu enclins à réagir et à financer la recherche sans certitudes...). A cette part d'imprécision des MCG à décrire la physique de l'atmosphère, s'ajoute celle de la prévision, du nombre d'êtres humains et leur mode de vie dans le futur. Or cette donnée va aussi conditionner les quantités de GES qui seront rejetées (transport, chauffage, utilisation des terres agricoles..., le tout dans un contexte de pénurie en énergies fossiles qui va aller en s'amplifiant).

Les résultats fournis par ces modèles ne doivent donc pas être interprétés sans un minimum de connaissances du principe de la modélisation ${ }^{11}$. Par exemple dans un MCG, les reliefs sont lissés à l'échelle des mailles du modèle; dans les régions d'upwelling/ courants froids la simulation des nuages stratiformes (à développement horizontal) semblent difficiles à simuler... Un minium de connaissances préalable est nécessaire pour comprendre et interpréter les "sorties de modèles» qui constituent les seuls outils permettant d'appréhender le futur climatique avec tout ce qu'il comporte d'incertitudes. Un MCG est donc un outil complexe, qui a pour objectif d'obtenir une représentation simplifiée du fonctionnement du système Terre-Océan-Atmosphère mais avec une part d'imprécision. 


\subsection{Le choix des échelles spatiales et temporelles}

30 Jusqu'à la fin du $\mathrm{XX}^{\mathrm{e}}$ siècle, la recherche fondamentale s'est surtout attachée à la modélisation du changement climatique sur de vastes territoires (les mailles des modèles les plus courants étaient de 2,5 de coté). Cependant, depuis une dizaine d'années, les modèles permettant d'accéder à des résolutions spatiales plus fines se multiplient. Les mailles de ces modèles se rapprochent de celles sur lesquelles il est possible d'envisager les conséquences du changement pour les Hommes (quartier de ville, terroir...).

31 Le passage de l'échelle des MCG jusqu'à l'échelle régionale est couramment désigné par le terme de "désagrégation » (figure 7). Ce passage peut se faire avec deux familles de méthodes qui peuvent être combinées entre elles (Giorgi, 2006) :

- la première fait appel à des techniques statistiques qui consistent à tenter de relier les variables de petite échelle (vaste surface) aux variables d'échelle régionale ou locale (grandes échelles des géographes) au moyen de modèles simples ou de fonctions ajustées grâce aux observations réalisées et à l'utilisation de Système d'Information Géographique (SIG) et de Modèle Numérique de Terrain (MNT) ;

- la deuxième fait appel à la régionalisation dynamique. Dans ce cas, on utilise un modèle de simulation du climat dont la résolution est plus fine que celle des modèles globaux.

Mais ces méthodes ont un coût de calcul élevé. Ce qui contraint à les utiliser soit à l'échelle globale, mais avec une résolution variable d'une région à l'autre ${ }^{12}$; soit sur un domaine limité, et contraint à ces limites par les données d'un MCG. Les modélisateurs désignent l'ensemble de ces outils à aire limitée sous le terme de Modèle Climatique Régional ou MCR. Les MCR permettent non seulement d'affiner spatialement les projections fournies par les MCG, mais aussi de corriger certains biais de ces derniers (Vigaud and al., 2009). Ce sont les résultats produits par ces MCR qui commencent à intéresser les chercheurs en Sciences Humaines, disciplines dans lesquelles les scientifiques ont la maîtrise d'outils permettant d'apporter des éléments de réponses aux questions que se pose la société sur le futur du climat et sur ses répercussions sociales et économiques. 


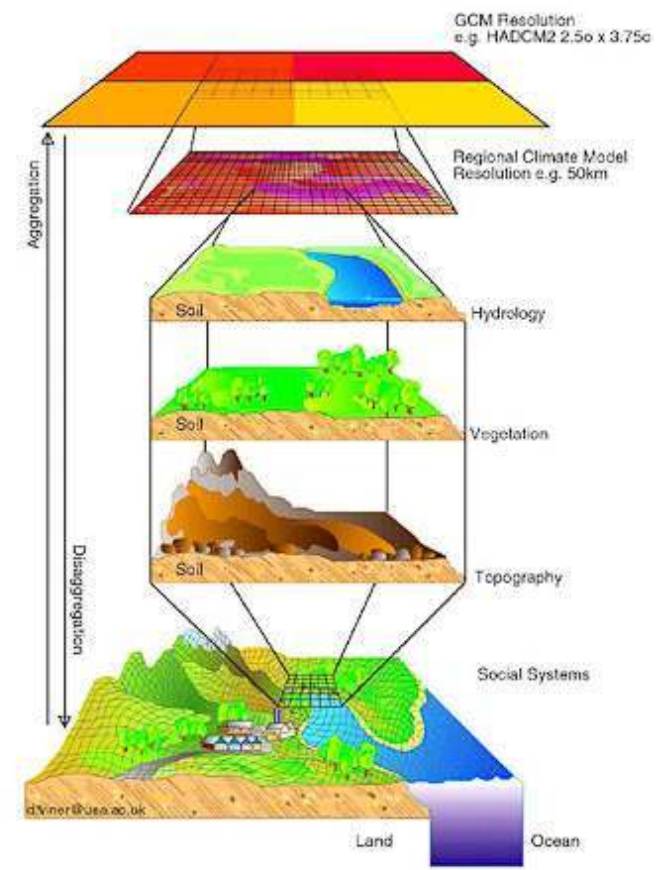

Source : d'après D. Viner, (C) Copyright 2000, Climatic Research Unit - University of East Anglia, Royaume-Uni.

Briche et al. (2009), à partir des données ARPEGE-Climat (Rétic), établissent des calendriers de probabilité pour évaluer les extrêmes thermiques - chauds estivaux et gélifs printaniers - en utilisant les sorties de ce modèle à l'échelle quotidienne jusqu'en 2100. Par exemple pour le scénario A1B (scénario « modéré»), des températures estivales supérieures à $35^{\circ} \mathrm{C}$ (température à partir de laquelle l'échaudage de la vigne peut se produire) devraient apparaître environ 2 à 3 jours par an à partir des années 2050 (figure 8) alors qu'elles sont quasi absentes aujourd'hui.... 
Figure 8 - Fréquences des températures journalières estivales (juin, juillet, août) au-delà de certains seuils et moyennes mobiles sur 10 ans, pour une surface de $50 \mathrm{~km}$ de coté couvrant la Champagne viticole (Marne) sur la période simulée 2001-2100, avec le scénario A1B

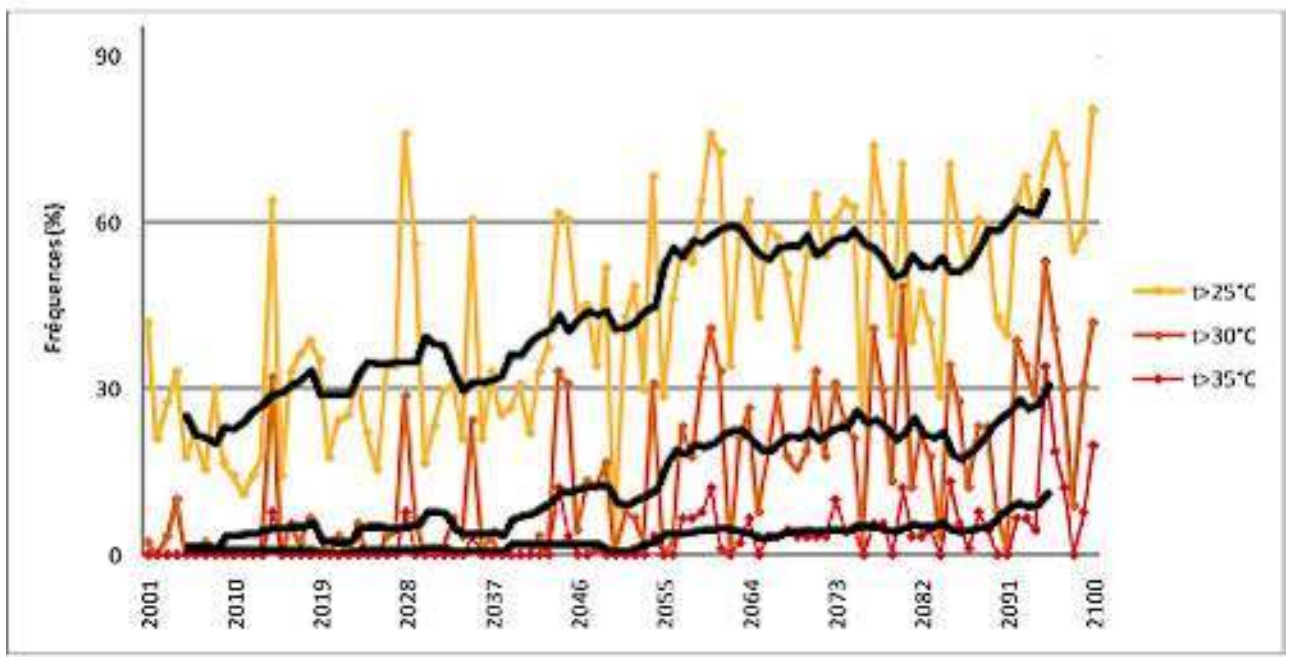

Source : Données Météo-France - CNRM. Briche et al., 2009

De même, Ullmann et Moron (2007) évaluent les modifications futures des surcotes marines dans le golfe du Lion. Ils montrent que la fréquence et l'intensité de ces phénomènes ne devraient pas augmenter significativement d'ici 2100, selon les résultats fournis par les scénarios A2 et B2. Cependant, ces surcotes se surimposeront à la hausse moyenne du niveau marin, inéluctablement croissant au moins dans l'absolu ${ }^{13} \mathrm{~A}$ la fin du $21^{\mathrm{e}}$ siècle, la fréquence des niveaux marins érosifs dans le Golfe du Lion (>40 cm NGF) pourrait dépasser $20 \%$ de l'hiver dans le scénario B2 et 30\% dans le scénario A2, pour les projections basses de la remontée du niveau marin moyen. Selon les projections hautes, le niveau aujourd'hui centennal (> $1 \mathrm{~m} \mathrm{NGF)}$ ) pourrait se produire plusieurs fois par hiver avec le scénario $\mathrm{A} 2$ et $\mathrm{B} 2 . .$.

Le changement climatique n'aura pas les mêmes répercussions environnementales, économiques et sociales dans tous les territoires et ces effets ne se feront pas ressentir partout avec la même ampleur ni pour les mêmes années. Ces inégalités territoriales focalisent déjà l'attention des chercheurs en Sciences humaines et sur certaines zones sensibles les analyses sont largement médiatisées : niveau des mers dans les îles coralliennes (Seychelles, Maldives...) ou les deltas surpeuplés (Brahmapoutre, Nil...); fonte des glaciers de montagne (réserve d'eau saisonnière pour les populations vivant en contrebas....) ou de la banquise (territoire de l'ours blanc...) ; déplacement des zones de production agricole (surtout lorsque ces cultures ont une forte valeur stratégique ou économique comme le blé ou la vigne...) ou la gestion sylvicole.

$\mathrm{Au}$ final, se pose la question de l'adaptation, au moins lorsqu'elle sera possible. Dans certains cas il faudra soit protéger les aménagements existants, soit accompagner les processus naturels par des interventions limitées; soit abandonner des espaces occupés et aménagés. Les enjeux ne sont pas toujours faciles à appréhender et les adaptations doivent être pensées à plusieurs échelles de temps et d'espace car le climat est en constante fluctuation et ignore les découpages administratifs. Les études sur les impacts $\mathrm{du}$ changement climatique doivent aussi intégrer le comportement des groupes humains car la mémoire des hommes est le plus souvent courte: il est bien difficile de faire 
admettre par exemple un risque de submersion par la mer à des populations qui souhaitent une vue sur la mer ou occuper des lieux connus et fréquentés parfois depuis plusieurs générations... Comment faire prendre conscience qu'une élévation de quelques $\mathrm{cm}$ du niveau de la mer pourra amplifier le risque de dommages, d'autant plus que nombre d'élus politiques, au-delà des discours de " parade », n'ont pas toujours la volonté de faire appliquer les lois existantes (loi littoral en France), ni de prendre des mesures de précaution inévitablement impopulaires?

\subsection{Un regard critique pour des réponses plus réalistes}

Le catastrophisme, la peur d'un futur climatique, a l'avantage de focaliser l'attention des médias et finalement d'attirer plus facilement les crédits de recherche dans les équipes qui le mettent en avant. A l'opposé, les scientifiques qui discutent de l'incertitude des modèles, qui montrent, connaissance du terrain et de la société à l'appui, que de nombreux facteurs autres que climatiques expliquent la fragilité de certains territoires et de certains groupes humains, sont eux moins médiatiques et in fine, disposent de moins de moyens pour leur recherche.

Pourtant, les géographes, spécialisés sur les questions environnementales, devraient s'impliquer plus dans les thématiques concernant le changement climatique aux échelles régionales que ce soit pour le paramétrage des données d'entrée des modèles (occupation du sol...) ou en aval de la modélisation numérique pour interpréter les «sorties de modèle ». Leurs travaux, tout en s'insérant dans des approches largement pluridisciplinaires, devront clairement montrer, sans concession, à la fois les aspects négatifs de ce changement en cours mais aussi les aspects positifs que ce soit pour certains territoires, certains groupes humains ou certains secteurs économiques. Cela devrait contribuer à renforcer des solidarités à toutes les échelles depuis celle de l'espace vécu par les individus jusqu'à l'échelle planétaire entre les pays riches principaux responsables de l'effet de serre additionnel et les pays pauvres qui en subissent les conséquences sans en être les principaux responsables. D'une manière plus générale, les Sciences Humaines doivent aussi montrer qu'il n'y a pas toujours de solution unique à un problème posé ou que celle qui, a priori, parait la plus évidente, n'est pas forcement celle qu'il faut retenir (l'épis sur une plage n'est pas toujours la solution...). Cet exemple du littoral peut être appliqué à d'autres types d'espaces comme la moyenne montagne, les terroirs agricoles ou forestiers, les milieux urbains... .

Le géographe qui travaille dans le domaine de l'environnement et des changements globaux a donc de passionnantes pistes de recherche à explorer lorsqu'il s'appuie sur un solide travail de terrain qui peut prendre des formes variées (quantification des processus, analyse des enjeux humains ...) et l'utilisation d'outils d'analyse (Géomatique, modélisation numérique...) tout en dialoguant avec les chercheurs d'autres disciplines au langage et aux méthodes de travail souvent assez différentes.

\section{Conclusion}

Après deux décennies marquées par la suprématie de la modélisation numérique, il est temps que les chercheurs des Sciences s'impliquent plus pleinement dans les recherches sur les conséquences du changement climatique. Les outils d'analyse et de simulation ont évolué, la demande sociétale se précise et les études sur l'adaptation au changement 
climatique ne pourront pas se contenter d'analyses combinant des résultats issus de modèles climatiques à ceux issus de modèles économiques pour tenter de se préparer à un futur climatique. Le chercheur qui souhaite se pencher sur ce sujet devra impérativement aller sur son terrain d'étude pour analyser les modes de vie de la population qui y vit et déterminer les enjeux. Il devra aussi prendre conscience puis expliquer sans détour la complexité et l'incertitude de ces résultats, tout particulièrement lorsqu'il s'agit d'évaluer les conséquences locales ou les conséquences liées aux phénomènes météorologiques extrêmes plus difficiles à simuler. Pour cela il faudra commencer par renforcer le dialogue entre les disciplines, au minimum pour mieux comprendre et interpréter les « sorties » de modèle climatique.

Les enjeux posés par le changement climatique sont importants et, même si son ampleur est difficile à préciser, la certitude de ce changement déjà en cours parait difficilement discutable. Si dans le passé l'homme s'est toujours adapté aux crises environnementales, il n'en demeure pas moins qu'aujourd'hui la question du climat, qui interfère avec celle de l'empreinte écologique, constitue l'une des priorités de la recherche.

\section{BIBLIOGRAPHIE}

Belia S, Douguédroit A. et Seguin B., 2008. Impact du réchauffement sur les étapes phénologiques du grenache et de la Syrah dans les Côtes-du-rhône méridionales et les Côtes de Provence (1976-2000). Actes des Journées de Climatologie de la commission de Climat et société du CNFG, Nantes, p. 45-54. http://134.59.38.8/ umr/climat/climat_societe/Nantes2008/3-AD_cd.pdf

Beltrando G. et Camberlin P. 1993. Interannual variability of rainfall in the Eastern Horn of Africa and indicators of atmospheric circulation. Int. J. of Climato., 13, p. 533-546. Résumé disponible: http://www3.interscience.wiley.com/journal/113491366/abstract

Beltrando G. \& Cadet D.L., 1990. Variabilité interannuelle de la petite saison des pluies en Afrique orientale : relations avec la circulation atmosphérique générale. Veille Climatique Satellitaire, 33, p. 19-36.

Bigot S., Laurent J.-P., Rome S. et Biron P.-E., 2006 : Mise en place d'un observatoire pour le suivi éco-climatique des Hauts Plateaux du Vercors. Actes du XIXe colloque de l'Association Internationale de Climatologie, Epernay, p. 125-130 Disponible : http://climato.be/aic/colloques/actes/ epernay2006_actes.pdf

Bodin X., 2007. Géodynamique du pergélisol de montagne : fonctionnement, distribution et évolution récente. L'exemple du massif du Combeynot (Hautes Alpes). Thèse de l'Université Paris-Diderot, $274 \mathrm{p}$; Site web : http://tel.archives-ouvertes.fr/docs/00/20/32/33/PDF/Bodin_these_2007.pdf Bois B., 2007. Ce que nous apprennent le climat récent et les observations phénologiques sur les effets du changement climatique en Gironde viticole. Actes de la 8ème Journée Technique du CIVB, 13 mars, Bordeaux, p. 72-80.

Bonnardot V., 1996. Le climat et la vigne en Bourgogne orientale. Thèse de Doctorat de l'Université de Bourgogne (Dijon), 236 p. 
Bonnefoy C., Quénol H., Barbeau G. et Madelin M., 2009. Analyse multiscalaire des températures dans le vignoble du Val de Loire. Actes du XXII colloque de l'Association Internationale de Climatologie (Cluj-Napoca) Geographia Technica. http://geografie.ubbcluj.ro:8010/AIC/ pdf/14_c._bonnefoy_analyse_multi_scalaire_des_temperatures_dans_le.pdf

Briche E., Beltrando G., Kergomard C. et Quénol H., 2009. Analyse comparative des sorties thermiques du modèle numérique ARPEGE-CLIMAT pour une simulation bioclimatique jusqu'à la fin du XXIème sicle sècle dans le vignoble de Champagne. Actes du colloque Théoquant, Besançon.

Camberlin P., 2004. Contributions à la compréhension de la variabilité pluviométrique multi-échelle en Afrique. HDR, 101 p., CRC - Université de Bourgogne, Dijon, 101 p.

Chabin J.-P, Madelin M. et Bonnefoy C., 2007. Les vignobles beaunois face au réchauffement climatique. Colloque Réchauffement climatique, quels impacts probables sur les vignobles? Chaire UNESCO, Dijon et Beaune, 28-30 mars, 13 p. http://www2.u-bourgogne.fr/chaireunescovinetculture/Actes\%20clima/Actes/Article_Pdf/Chabin.pdf

Chenet M. et Roussel E., 2008 : Changement climatique et atrophie des masses glaciaires : quelles mutations pour les paysages islandais? EchoGéo, Numéro $4 \mid 2008$, [En ligne], mis en ligne le 21 février 2008. http://echogeo.revues.org/index1883.html.

Douguédroit A. et Bridier S., 2008. Sur la détermination de la date du début de la tendance actuelle au réchauffement. Actes $\mathrm{du} \mathrm{XXI}^{\mathrm{e}}$ colloque de l'Association Internationale de Climatologie (Montpellier), p. 201-206. http://climato.be/aic/colloques/actes/montpellier2008_actes.pdf

Duchêne E. et Schneider Ch., 2007. Conséquences écophysiologiques des évolutions climatiques au cours du cycle de développement de la vigne en Alsace. Colloque Réchauffement climatique, quels impacts probables sur les vignobles? Chaire UNESCO, Dijon et Beaune, 28-30 mars. http://www2.ubourgogne.fr/chaireunesco-vinetculture/Actes\%20clima/Actes/Article_Pdf/Duchene.pdf

Fort M., Cossart E., Deline P. Dzikowski M., Nicoud G., Ravanel L., Schoeneich P. et Wassmer P., 2009. Geomorphic impacts of large and rapid mass movements : a review. Géomorphologie, 1, p. 47-63

Giorgi F., 2006. Regional climate modelling: status and perspectives. J. Phys., 139, 101-118 DOI: 10.1051/jp4:2006139008

IPCC, 2007. Climate Change: The Physical Science Basis. Summary for Policymakers. Contribution of the Working Group I to the Fourth Assessment of the Intergovernmental Panel on Climate Change, 18 p. http://www.ipcc.ch/pdf/assessment-report/ar4/wg1/ar4-wg1-spm.pdf

Jones G., Duchene, E., Tomasi, D., Yuste J., Braslavska O., Schultz H., Martinez, C., Boso S., Langellier F., Perruchot C. et Guimberteau G., 2005. Actes du colloque Changes in European winegrape phenology and relationships with climate, GESCO 2005, Allemagne, Vol. 1, p. 55-62.

Kergomard C., 2005. L'Arctique face au changement climatique : observations, modèles, impacts. Actes des Journées de Climatologie du CNFG: le changement climatique, Dijon, 17-19 mars, p. 35-51. http://134.59.38.8/ umr/climat/climat_societe/Dijon2005/3CKcd.pdf

Le Clainche Y., Braconnot P.,Marti O., Joussaume S., Dufresne J.-L. and Filiberti M.-A., 2001.The role of sea ice thermodynamics in the Northern Hemisphere climate as simulated by a global coupled ocean-atmosphere model. Note scientifique IPSL du pôle de modélisation du climat $\mathrm{N}^{\circ}$ 21, http://www.ipsl.jussieu.fr/poles/Modelisation/NotesScience/note21.pdf

Levitus S. J., Antonov J. \& Boyer T., 2005. Warming of the world ocean, 1955-2003. Geophys. Res. Letters, 32, L02604, doi:10.1029/2004GL021592. ftp://ftp.nodc.noaa.gov/pub/data.nodc/woa/ PUBLICATIONS/GL023112.pdf 
Lombard A., 2007. Les variations actuelles du niveau de la mer : observations et causes climatiques. La Météorologie, 59, p. 13-21. http://www.smf.asso.fr/Ressources/Lombard59.pdf

Loubier J.-C., 2007. Changement climatique et domaines skiables: simulation en Savoie et HauteSavoie à l'horizon 2015. Mappemonde, 85. http://mappemonde.mgm.fr/num13/articles/ art07103.html

Pachauri R.K. and Reisinger, A. (Eds.), 2007. Contribution of Working Groups I, II and III to the Fourth Assessment Report of the Intergovernmental Panel on Climate Change. IPCC, Geneva, Switzerland, p. 104 http://www.ipcc.ch/pdf/assessment-report/ar4/syr/ar4_syr_fr.pdf

PIGB-PMRC, 2002. Lettre $n^{\circ} 14$ Programme International Géosphère Biosphère-Programme Mondial de Recherches sur le Climat. http://www.cnrs.fr/cw/dossiers/dosclim/biblio/ pigb14/11_rechauffement.htm

Rasmusson and Carpenter, 1982. Variations in tropical sea surface temperature and surface wind fields associated with the Sothern Oscillation El Nino. Mon. Wea. Rev., 110, p. 354-384. http:// www-pord.ucsd.edu/ ltalley/sio219/rasmusson_carpenter_mwr1982.pdf

Seguin B., 2006. Le réchauffement récent attesté par les impacts observés sur les écosystèmes. Actes du XIX ${ }^{e}$ colloque de l'Association Internationale de Climatologie Les risques liés au temps et au climat, Épernay, p. 27-33. http://climato.be/aic/colloques/actes/epernay2006_actes.pdf

Somot S., 2005. Régionalisation des scénarios de changement climatique. Environnement, Risques \& Santé, 4, p. 89-94. http://www.john-libbey-eurotext.fr/fr/revues/sante_pub/ers/edocs/00/04/0B/69/article.phtml

Ullmann A., 2007. Niveaux marins extrêmes dans le golfe du Lion : variabilité au 21ème siècle dans les scénarios A2 et B2. Actes des Journées de Climatologie du CNFG : mesures et modèles, Grenoble, 22-23 mars , p. 109-123. http://umrespace.unice.fr/public_html/umr/spip/spip.php?article137

Vigaud N., Roucou P., Fontaine B., Sijikumar S. \& Tyteca S., 2009. WRF/ARPEGE-CLIMAT simulated climate trends over West Africa. Climate Dynamics, 10. Résumé disponible : http:// www.springerlink.com/content/mr3w84w338514513/fulltext.pdf?page=1

Walther G-R, Post E., Convey P., Menzel A., Parmesan P., Beebee T JC, Fromentin J-M, HoeghGuldberg O. \& Bairlein F., 2005. Ecological response to recent climate change. Nature, 416, p. 389-395, doi:10.1038/416389a.

\section{NOTES}

1. Voir par exemple le rapport de synthèse réalisé pour l'International Panel of Climatic Change - IPCC - de Pachauri \& Reisinger (2007), intégrant les résultats de nombreuses publications.

2. Voir par exemple PIGB-PMRC (2002) pour les modèles globaux ou plus récemment Somot (2005) pour les modèles aux échelles régionales.

3. Ces derniers ont un rôle inverse car ils contribuent pour l'essentiel au refroidissement de l'atmosphère, mais dans une proportion moindre que les GES.

4. Exemple : l'augmentation de la réflexion sur les surfaces laissées sans végétation une partie de l'année est en moyenne planétaire à l'origine d'une diminution saisonnière de la quantité d'énergie absorbée en surface.

5. Exemple: lorsque le taux de couverture nuageuse augmente, il y a plus de réflexion du rayonnement solaire et donc in fine moins d'énergie qui est absorbée par l'atmosphère et la surface de la planète. 
6. Les autres types d'indicateurs du changement, tels que l'augmentation du rythme de disparition des espèces, la dégradation parfois irréversible, au moins sur le moyen terme, de certains milieux physiques ou l'acidification des océans ne sont pas abordés dans cet article.

7. Travaux dans le cadre du Programme National d'Etude de la Dynamique des Climats des années 1980, pour ne citer qu'un des plus anciens.

8. C'est le cas des glaciers de Franz Josef en Nouvelle-Zélande qui, implantés sur des pentes raides, ont été très réactifs aux petits changements de bilan de masse des années 90 .

9. Même si, bien évidemment, d'autres phénomènes océano-atmosphérique peuvent aussi intervenir dans la variabilité climatique.

10. Une période de réchauffement de l'air entraîne plus de fonte de glace sous les hautes latitudes, il y a donc plus d'eau douce en surface de l'océan, ce qui se traduit par moins de «descente » de cette eau moins salée et donc moins dense dans les fonds marins. Cela limite les transferts d'eau moins froide par les courants de surface et finalement il y a moins de transfert de chaleur par les courants atmosphériques.

11. Or les détails de la modélisation sont beaucoup plus complexes et peu de scientifiques en ont une connaissance parfaitement exhaustive.

12. Par exemple la dimension de la maille peut-être différente suivant qu'elle renseigne sur l'océan ou sur une région de montagne..., ce qui permet de mieux décrire une région particulière de la planète.

13. Car il peut y avoir simultanément subsidence du continent qui masque partiellement l'élévation du niveau moyen de la mer.

\section{RÉSUMÉS}

En France, sur le sujet du changement climatique et quel que soit son origine, les géographes climatologues se sont majoritairement intéressés à la description et l'explication de la variabilité du climat à diverses échelles de temps et d'espace sur des périodes écoulées. Ils se sont moins investis sur les formes d'adaptations tenant compte des résultats des simulations du climat futur, tel qu'il est envisagé à partir des calculs obtenus par les modèles numériques du climat. Cette frilosité s'explique en partie par le manque de précision et de fiabilité accordé aux résultats produits par ces modèles, aux échelles des territoires occupés par les sociétés humaines, mais aussi parce que les travaux de géographes montrent depuis longtemps que les sociétés humaines - indépendamment du changement climatique sous l'effet des gaz à effet de serre additionnels ont une part directe dans bien des catastrophes qui les affectent (construction dans des zones inondables, production agricole dans des régions où la pluviométrie peut être insuffisante certaines années...). Les géographes-climatologues, commencent à utiliser des nouvelles sorties de modèle à des échelles plus opérationnelles pour proposer des formes d'adaptations spécifiques à chaque territoire et à chaque secteur économique. Ces recherches prospectives sont réalisées en tenant compte à la fois de la part d'incertitude des résultats produits par des modèles numériques, mais aussi des particularités des groupes sociaux et des milieux physiques dans lesquels s'inscrivent ces changements. Cela est un préalable nécessaire à toutes formes de réflexion approfondies sur l'identification des facteurs d'adaptation.

In France, on the topic of climate change, the geographer climatologists are focused on the description and explanation of climate variability at various temporal and spatial scales on last 
period. They are less interested in the forms of adaptation considering results of future climate simulations, as envisaged by numerical climate models. This discretion is explained by the lack of climate model precision at the scale of territories occupied by human societies, but also because geographer research have shown since long time that human societies - with their involvement in additional greenhouse effect- have a direct effect in many disasters, which affect them (construction in flood plains, agricultural production in regions where rainfall can be insufficient sometimes...).Geographer climatologists can use new models at more operational scales to purpose specific adaptation for each area and each economical sector. These investigations integrate numerical model simulation uncertainties, but also social group and physical environment particularities concerning by these changes. This is the condition for reflection about the identification of adaptation factors

\section{INDEX}

Mots-clés : adaptation, changement climatique, dynamique glaciaire, Modèle Climatique Régional (MCR), Modèle de Circulation Générale (MCG), viticulture

Keywords : adaptation, climate change, General Circulation Model (GCM), ice dynamics, Regional Climate Model (RCM), wine-growing

\section{AUTEUR}

\section{GÉRARD BELTRANDO}

Gérard Beltrando est climatologue, Professeur de Géographie à l'Université de Paris-Diderot et membre de l'UMR PRODIG. Il est actuellement détaché au CNRS à l'UMR LETG (Equipe COSTEL) pour travailler sur les impacts du changement climatique aux échelles régionales beltrando@univ-paris-diderot.fr 